\title{
Completely New Gravitational Physics: The Ingenious Outside-Inside Centrifuge Mechanism of Gravity Clarified
}

\author{
Jacob Schaf \\ Universidade Federal do Rio Grande do Sul (UFRGS), Instituto de Fsica, Porto Alegre-RS, Brazil \\ Email: schaf@if.ufrgs.br
}

How to cite this paper: Schaf, J. (2019) Completely New Gravitational Physics: The Ingenious Outside-Inside Centrifuge Mechanism of Gravity Clarified. Journal of Modern Physics, 10, 225-255.

https://doi.org/10.4236/jmp.2019.103017

Received: November 7, 2018

Accepted: March 4, 2019

Published: March 7, 2019

Copyright (อ 2019 by author(s) and Scientific Research Publishing Inc. This work is licensed under the Creative Commons Attribution International License (CC BY 4.0).

http://creativecommons.org/licenses/by/4.0/

\section{(c) (i) Open Access}

\begin{abstract}
The present work implements the idea that gravity is not a fundamental force and that the observed gravitational dynamics is the result of inertial motions within a Keplerian velocity field of the Higgs Quantum Space (HQS), giving mass and ruling the inertial motions of matter-energy. The Higgs theory introduces profound changes in the current view about the nature of empty space. It introduces the idea that a real quantum fluid medium, filling up the whole of space, gives mass to the elementary particles by the Higgs mechanism, an effect analogous to the Meissner effect, giving mass to the photons within superconductors. This HQS necessarily governs the inertial motion of matter-energy and is the locally ultimate reference for rest and for motions. The HQS materializes the local Lorentz frames (LFs), turning them into local proper LFs, intrinsically stationary with respect to the local moving HQS. This HQS also necessarily is responsible for the gravitational dynamics; because it is mass that creates the gravitational fields. The observed absence of the gravitational slowing of the GPS clocks by the solar field and the absence of light anisotropy with respect to the moving earth are both obvious signatures of the true physical mechanism of gravity. These observations demonstrate that the HQS is circulating round the sun and round earth according to Keplerian velocity fields $(G M / r)^{1 / 2}$, closely consistent respectively with the planetary motions and the orbital motion of the Moon. In this Keplerian velocity field the planets are closely stationary with respect to the local HQS and carried by the moving HQS round the sun without the need of a central force field. The Keplerian velocity field of the HQS is the only possible imaginable mechanism able to give rise to the ingenious outside-inside centrifuge mechanism of gravity that creates a central field of centrifugal forces toward the gravitational center on all matter bodies not moving strictly along direct circular equatorial orbits. The Keplerian velocity field of the HQS is shown to
\end{abstract}


appropriately create all the observed effects of the gravitational fields on matter, on light and on clocks.

\section{Keywords}

Fundamental Physics, Higgs Theory, Gravitational Physics, Gravitational Dynamics, Gravitational Effects

\section{Introduction}

In his General Theory of Relativity (GR) [1] [2] Einstein has replaced the Newtonian gravitation by a completely new theory of gravitation, in which no gravitational forces are needed. In order to explain the observed gravitational dynamics, he assumed that the gravitational sources curve the geometry of the four-dimensional spacetime in their neighborhood and that, in this curved spacetime, the orbital motion of the matter bodies follows geodesic paths, governed by a generalized principle of inertia. According to this principle, the gravitational pull on earth is equivalent to an inertial pull and any locally non-accelerated body is a proper reference. He described this spacetime curvature by his famous field equations for the four-dimensional metric tensor $g_{\mu v}$ :

$$
G_{\mu v} \equiv R_{\mu v}-\frac{1}{2} g_{\mu v} R=8 \pi G T_{\mu v}
$$

where $R_{\mu v}$ is the Ricci curvature tensor, $R$ is the scalar curvature, $G$ is the gravitational constant and $T_{\mu \nu}$ is the stress-energy tensor of the gravitatonal source. In the neighborhood of a spherically symmetric gravitational source, the metric of this curved spacetime is characterized by the invariant length of the four-dimensional line element $\mathrm{d} s$. For sufficiently weak gravitational fields, it is described by:

$$
\mathrm{d} s^{2} \approx\left[1-\frac{2 U}{c^{2}}\right]^{-1} \mathrm{~d} r_{0}^{2}+r^{2} \mathrm{~d} w^{2}-c^{2}\left[1-\frac{2 U}{c^{2}}\right] \mathrm{d} t_{0}^{2}
$$

The coefficients $\left(1-\frac{2 U}{c^{2}}\right)^{-1}$ and $-c^{2}\left(1-2 U / c^{2}\right)$ are respectively the diagonal $g_{11}$ and $g_{44}$ components of the Schwarzschild metric tensor, where $c$ is the velocity of light in free empty space and $2 U=2 G M / r$ is the square of the local escape velocity from the gravitational field.

The last term in Equation (2) expresses the gravitational time dilation as viewed by an external observer. There $\mathrm{d} t_{0}$ is an infinitesimal time interval in the absence of gravity. From the view of an external observer, the rate of a clock within a gravitational field runs slowly, which can be expressed as a slower effective velocity $\left(c^{2}-2 G M / r\right)^{1 / 2}$ along the time axis. In the first term of Equation (2), $\mathrm{d} r_{0}$ is a radial distance in the absence of gravity. The time interval $\mathrm{d} t$ for a light pulse to travel a distance $\mathrm{d} r$ along the radial coordinate can be written as $\mathrm{d} t=\mathrm{d} r / c=\mathrm{d} r_{0} /\left(c^{2}-2 U\right)^{1 / 2}$, where $c^{\prime}=\left(c^{2}-2 G M / r\right)^{1 / 2}$ again is the effective 
velocity of light, however along the radial coordinate. From the view of an external observer, the effective velocity of light $c^{\prime}$ and the rate of a clock both gradually reduce toward the gravitational center and fall to zero at the event horizon of a black hole.

According to the last term in Equation (2), clocks, stationary within a gravitational field, run slowly because the period of the time standard $T$, by which the clock counts time, is longer by a factor $\left(1-2 U / c^{2}\right)^{-1 / 2}$, given by:

$$
T(r)=T_{0}\left(1-2 U / c^{2}\right)^{-1 / 2}
$$

where $T_{0}$ is the time period for $(U=0)$.

\section{Actually Several Predictions of GR Discord from Observations}

Atomic clocks, stationary within the earth's gravitational field, confirm exactly the gravitational slowing predicted by GR. However, recent experimental observations have revealed that the GPS clocks, moving with earth round the sun, do not show the gravitational slowing by the solar field. As the escape velocity (from the solar field) does not depend on the orbital velocity, this observation runs into conflict with Equation (3). It demonstrates that the orbital velocity of earth cancels locally the effects of the solar gravitational field, restoring locally the conditions of a proper reference.

Current theories [3] explain the absence of the gravitational slowing of the GPS clocks by the solar field in terms of the principle of equivalence. Accordingly, the local Lorentz frame (LF) of earth is a local proper LF in which the orbiting earth as well as the GPS clocks, moving with it, are stationary. It is alleged that these clocks are free-falling with earth in the solar field and that this orbital motion cancels locally all the effects of the solar gravitational field. According to Einstein, in a free-falling elevator the effects of gravity are locally canceled and the conditions of a proper reference are locally restored. However, consider elevators falling from all different altitudes, at velocities from zero to the local escape velocity. How can all these mutually moving elevators at a same given point be proper references? Moreover, according to this view, if the free-fall of the references is stopped, all clocks in them begin to run slow according to Equation (3) and the path of light becomes bent. The upward force, stopping the free-fall, seems to be the cause of the gravitational slowing. However, Muon decay in Cyclotrons [4] demonstrates that accelerations up to $10^{19} \mathrm{~m} / \mathrm{sec}^{2}$ do not give rise to any effect on the mean life-time of Muons. Only velocity causes the time dilation. In fact, the same GPS clocks too are moving with the GPS satellites along circular orbits round earth, which, according to the above interpretation of the principle of equivalence, too should cancel the gravitational slowing by the earth's field on them. Nevertheless, these same theories [3] include, for the time rate of the GPS clocks, the gravitational slowing by the earth's field of $1.67 \times 10^{-10} \mathrm{sec} / \mathrm{sec}$, which clearly is at odds with the previous assumptions in the 
case of motions round the sun.

The fundamental question to be answered here is: Are all these relativistic effects simply perspectives, depending on the relative velocity of the observer as assumed in the theory of relativity, or are they real and do not depend on the observer's kinematical state? The present work will take the second alternative. The Higgs theory introduces fundamental changes in Einstein's view about the nature of empty space. It introduces the idea that a real quantum fluid medium, filling up the whole of space, gives mass to the elementary particles by the Higgs mechanism. This Higgs Quantum Space (HQS) is much more than simply a local reference for rest and for motions. It necessarily rules and governs the inertial motions of matter-energy and is the local ultimate (local absolute) reference for rest and for motions.

The present work adopts the view of the Higgs theory. The Higgs theory, besides disclosing the origin of the inertial mass, opens the new way to understand the origin of the gravitational dynamics. The HQS materializes the local Lorentz Frames (LFs), turning them into local proper LFs, intrinsically stationary with respect to the local HQS. Only motions with respect to the local HQS (proper $L F S$ ) and not relative velocities are the origin of all the effects of motion. From this viewpoint the effects of motion are real, depending on the velocity with respect to the local HQS and not on the relative velocity of the observer. Within this new scenario, the absence of the solar gravitational slowing of the GPS clocks and the absence of the light anisotropy with respect to earth demonstrate that earth is stationary with respect to the local HQS (proper LFs). This can make a sense only if the HQS is moving round the sun consistently with the orbital motion of the planets. From this viewpoint, the only possible and sensible conclusion from the observations is that not every circular orbital motion cancels locally all the effects of the gravitational field. Only motions along direct circular equatorial orbits (planetary motions) cancel all the effects of the solar gravitational field. All the other orbital motions, including retrograde circular equatorial orbital motions and free-falling references are not proper LFs and do not cancel locally all the effects of the gravitational field. They cancel only the gravitational pull. The point here is not putting in doubt the correctness of the estimate of the gravitational slowing of the GPS clocks by the current theories, however showing that the explanation in terms of the principle of equivalence in the case of the solar field and in the case of the earth's field are not consistent with each other. They hence cannot be true explanations.

The explanation in terms of the free-falling inertial references is an Aristotelian like explanation, according to which bodies fall because their inertial references fall (they fall because they fall). However, why do the local inertial references fall? The model of the free-falling inertial references has another much more serious shortcoming. It cannot give rise to a gravitational pull on a stationary body. It cannot because the velocity of Einstein's free-falling inertial references at the local escape velocity is fixed at each point $\left(r_{0}, \theta_{0}, \phi_{0}\right)$ of space. 
Moreover, any such inertial reference is valid at only one spatial point. Locally, the acceleration of the free-fall velocity, seen by a stationary body, is zero $\left(\mathrm{d} v /\left.\mathrm{d} t\right|_{r_{0}}=0\right)$. Obviously, a velocity constant in time and direction fixed in space cannot give rise to an inertial pull. Therefore, a matter body, brought to rest at any fixed point in a gravitational field and then released, should stay in equilibrium there. Any perturbation however would initiate a runaway departure, upward or downward. In order to enable Einstein's model of the free-falling inertial references to produce a gravitational pull, it would be necessary that the local free-fall velocity, at any fixed position $r_{0}$, be given by $\mathrm{d} v /\left.\mathrm{d} t\right|_{r_{0}}=g\left(r_{0}\right)$. However, then the free-fall velocity would rapidly increase beyond the velocity of light, which clearly cannot be reasonable.

In a gravitational field, in which the gravitational dynamics is the result of purely inertial motions (as required by GR), all matter bodies, not acted on by an external force, are free bodies. Hence, the real vertically upward forces on such free bodies, necessary to prevent their free-fall on earth and acting without producing any vertically upward velocity, can obviously be only of a centripetal nature. Centripetal forces are real and act in a direction normal to the instantaneous velocity vector of the moving body. If the velocity of such a body is zero, the necessary centripetal force too is zero. Centripetal forces cause only a continuous change of the direction of the body's velocity vector, letting its magnitude perfectly constant. They produce no velocity along their instantaneous direction. In a gravitational field, the reaction force, opposite to the real (upward) centripetal force, is a downward centrifugal pull, which is a fictitious force exactly as Einstein wanted.

In order to implement, on earth, the scenario of the centrifugal pull toward the earth's gravitational center on stationary matter bodies, it is necessary that each stationary matter particle be implicitly moving along a circular path round an over-head axis. Such an implicit motion can be created only if a local spatial medium, ruling the inertial motion of matter-energy and being the local ultimate (local absolute) reference for rest and for motions is moving non-uniformly in the ordinary space along the horizontal direction oppositely to the implicit velocity of the body. The Higgs theory introduces exactly such a physical space, a quantum fluid medium, giving inertial mass to the elementary particles and thus governing their inertial motion. If the horizontal velocity of this spatial medium increases toward the gravitational center, the distribution of velocity through any infinitesimal region in the plane normal to the implicit velocity of the particle (plane of the wave fronts of its matter wave) corresponds to rotation round an over-head axis. In this scenario, the local inertial reference too will be rotating synchronously round the same over-head axis. The idea of a rotating reference being an IR may seem stupid. However, if it is this physical space, governing the inertial motion of matter-energy that is itself locally so rotating, this is all what is real and senseful. In this scenario, any local reference, not rotating in the ordinary space, is physically a non-inertial reference implicitly rotating oppositely 
round the same overhead axis. A body, stationary in the ordinary space within the velocity field (gravitational field) will implicitly be moving, within the local true IR, along a circular path round the same over-head axis. However, this it can do only under an adequate upward centripetal force. The motion of such a body is implicit, because it cannot be described in the ordinary space. It however is physically real and has all the usual consequences of motion.

The next Section 3 briefly discusses the nature of the quantum fluids, their relevant physical properties, the Higgs mechanism giving mass to the elementary particles, the coupling of quantum fluids with certain fields giving rise to dynamics etc. Section 4 describes details of the ingenious physical mechanism of gravity. Section 5 discusses the predictions of the Special and General theories of relativity within the scenario of the HQS dynamics. Section 6 sets up and solves the differential equation describing the gravitational dynamics in the solar system and on earth. Section 7 highlights the symmetry with orbital motions. Finally Section 8 describes a large number of experimental observations that all are perfectly and adequately produced by the HQS dynamics gravitational mechanism.

\section{The Higgs Quantum Space and the Foundations of the Gravitational Physics}

According to the gauge theories, all the elementary particles (field quanta) are originally massless and moving at the velocity of light. However, in fact most elementary particles are observed to have rest masses. Explaining the origin of this rest mass was one of the hardest problems of fundamental physics to solve. The first clue of the physical origin of the inertial mass was found 1963 by Anderson in superconductivity. [5] The superconducting condensate is a charged quantum fluid of paired conduction electrons (zero spin) condensed into a phase coherent quantum state. Electromagnetic (EM) fields couple to the superconducting (SC) condensate and the vector potential, associated with a changing magnetic field, creates electric fields that cause phase displacements (phase gradients), generating screening velocity fields of the SC-condensate. The created phase disorder elevates the energy of the SC-condensate. These screening currents confine the magnetic field into quantized fluxons or expel it out from the superconductor, which is the Meissner effect. [6] The Meissner effect takes place, because the confinement and expulsion of the field lowers the energy of the SC-condensate. Anderson discovered that gauge transformations of the SC order parameter, in the presence of a magnetic field, reveal mass terms of the EM field quanta (photons). However, the gauge transformations obviously do not create the mass of the photons. They only reveal it on testing the mobility of the photons within the superconductor. Hypothetically, accelerating a photon within a superconductor (by an external force) involves the creation of additional phase gradients and an additional velocity field of the SC-condensate. This additional velocity field too is perfectly persistent, which adds additional inertial mass to 
the photon.

Condensation of bosons (or pseudo-bosons $\mathrm{He}_{4}$ and or Cooper electron pairs) into a phase coherent state takes place at low temperatures, when the Bose-Einstein (BE) phase correlation between the boson wave-functions is sufficiently strong to overcome the thermal fluctuations and cause breakdown of the $U(1)$ symmetry of the bosons. Along a second order phase transition the bosons can lower their energy by condensing into a phase coherent macroscopic quantum state, liberating the corresponding amount of energy. This second order phase transition involves no latent heat and the energy is liberated gradually until zero temperature. In the macroscopic quantum state, the bosons become identical, completely entangled and indistinguishable. Their uncertainty in momentum and energy tend to zero, while the uncertainty in position and in time tend to be large (very large in the Higgs condensate). The condensate usually is described by the complex macroscopic Ginsburg-Landau like order parameter $\Phi(r, \theta)=\phi(r) \mathrm{e}^{i \theta}$, where $\phi(r)$ is an amplitude and $\mathrm{e}^{i \theta}$ is a phase factor. [7] The condensate responds only to specific fields and the response always is coollective, involving all the bosons of the condensate, through the order parameter.

The $\mathrm{BE}$ correlation gives rise to a negative potential energy (bonding) term, the value of which increases linearly with the condensate density $\rho=\Phi^{*} \Phi$. BE phase correlation leads to phase coherence of the boson wave-functions, lowering their energy, an effect analogous to the phase correlation between atomic orbitals forming the molecular bonds. Another positive potential energy (anti-bonding) term arises from repulsive core interaction between the bosons, that increases with the squared density $\rho^{2}$ and prevents collapse of the boson system. The effective potential $U(\rho)$ is mainly the sum of these two terms that has the form:

$$
U(\rho)=-n\left(\Phi^{*} \Phi\right)+m\left(\Phi^{*} \Phi\right)^{2}
$$

where the value of the negative coefficient $(-n)$ of the bonding term is considerably larger than the positive coefficient $(+m)$ of the anti-bonding term. Therefore the minimum of the effective potential energy occurs, not as usually for $\rho=0$, however for a finite value $\Phi^{*} \Phi=n / 2 m$, which is known as a non-zero vacuum expectation value. This effective potential is intrinsically highly homogeneous throughout the volume of the condensate. Quantum fluids have zero viscosity, are totally frictionless and perfectly conservative up to a well-defined velocity gradient. They couple only to very specific fields, which turns their presence extremely difficult to perceive.

The behavior of quantum fluids differs radically from classical fluids and even from perfect classical fluids. They really challenge commonsense. The fundamental characteristic of the quantum fluids is that their flow cannot be described in terms of positions as a function of time, because the wave function of each boson of the condensate already has non-zero amplitude in the whole volume. This makes their presence practically imperceptible. Consider a river of super-fluid 
helium. Like any river, let it not move from place. All possible physical tests to detect motion of the fluid in the river would fail. Imagine the river reaches a power station. The super-fluid would flow through the turbines without driving them at all.

The Higgs theory [8] [9] introduces fundamental changes in Einstein's view about the nature of empty space (vacuum), about the meaning of motions and about the nature of the gravitational physics. It introduces the idea that a scalar field, a real quantum fluid medium of zero spin particles, with properties closely analogous to the SC-condensate, fills up the whole of space. Breakdown of the electroweak symmetry and subsequent spontaneous breakdown to the $U(1)$ symmetry of the weak force doublet results in the Higgs quantum condensate. In the actual low temperature of the universe, this Higgs Quantum Space (HQS) is an extremely stable, rigid and powerful quantum fluid medium, ruled by the principles of quantum physics and, likewise the SC-condensate, it can be described by a complex Ginsburg-Landau like order parameter $\Phi(r, \theta)=\phi(r) \mathrm{e}^{i \theta}$. However, while the SC-condensate is stabilized by an energy gap of only about 1 $\mathrm{meV}$ per particle and condenses only at very low temperatures, the HQS is stabilized by a huge energy gap. According to the Glashow-Weinberg-Salam electroweak model, [10] [11] the energy gap achieves $200 \mathrm{GeV},\left(10^{14}\right.$ times larger than the energy gap of the usual superfluids and superconducting condensates) and condenses at $10^{15} \mathrm{~K}$. This enormous energy gap very strongly suppresses the quantum fluctuations and the zero-point energies of the various nuclear force fields, thereby leading to vacuum energy densities consistent with the observed values [12].

The HQS is much more than simply a local reference for rest and for motions. It is responsible for the mechanical properties of matter-energy and hence necessarily governs the inertial motion of the elementary particles. It also necessarily is the locally ultimate (local absolute) reference for rest and for motions of matter-energy. However, if the HQS is responsible for the mass and the mechanical properties of the elementary particles, it necessarily too is responsible for the gravitational fields, because it is mass that creates the gravitational fields.

Likewise the SC-condensate confines the EM field by the Meissner effect [6] down to $10^{-7} \mathrm{~m}$, quantizing it and giving inertial mass to the photons within superconductors, the Higgs quantum condensate or Higgs Quantum Space (HQS) confines the weak and strong nuclear foce fields down to $10^{-19} \mathrm{~m}$, giving large inertial masses to the vector bosons of the weak force field by the Higgs mechanism as well as to all the elementary particles, porting hyper-charges, by an indirect Higgs mechanism. [8] [9] Also, analogously as the motion of the EM field quanta within the SC-condensate involves additional mass, motion of the elementary particles within the local HQS, too involves additional phase perturbation of the Higgs order parameter, additional velocity fields of the HQS, additional excitation energy, additional mass-energy and additional elevation of the energy of the Higgs condensate. The role of the HQS in the life of our universe is 
absolutely vital. Without it, no laws of motion would exist and the universe, as we know it, simply could not exist.

Many authors insist that coupling of the nuclear fields to the HQS creates only a small part of the inertial mass of the elementary particles. This may well be true in a static situation. They justify this by saying that most of the mass of the elementary particles is relativistic mass, created by the high velocities of the internal confined constituents (quarks) of the real particles. This too can be true. It however is not relative velocity that causes this additional mass, but the velocity of these constituents with respect to the local HQS. Analogously as in the case of EM field quanta in superconductors, the motion of these constituents involves additional phase displacements, additional screening velocity fields of the Higgs condensate and additional energy. Moreover, all these additional are perfectly persistent.

In the case of the empty space, which is essentially the Higgs condensate in its ground state (without excitations), no external action can be invoked to explain the origin of the motions or changes of motion of isolated matter particles. Usual quantum fluids are well known to be totally in viscid, non-dissipative and intrinsically perfectly conservative. It also is well-known that the total momentum of any isolated particle or system of interacting particles is rigorously conserved. This demonstrates that the HQS does not itself manifest inertial mass and momentum effects. Therefore, any motion, once excited, becomes indefinitely persistent and can only be stopped by an opposite action of the same mechanism (field) creating the motion. This indefinite persistence is the very origin of the inertial behavior and the conservation of the momentum of isolated particles or particle systems. Also, due to the very high energy gap, the HQS too strongly suppresses quantum fluctuations, the creation of virtual particles and zero-point energies. It only intermediates, without loss, the effects of the various force fields between the elementary particles or particle systems. If the HQS itself could exhibit mass effects, the momentum as well as the other characteristic parameters of an isolated particle would not be conserved. However, in the real world, particles truly isolated from other particles do not exist. The fundamental requisite to create an elementary particle is a sufficient amount of energy. All the other characteristic parameters of the elementary particles were created from nothing but energy in the beginning of the universe, according to symmetries and laws of conservation. All together these parameters add up to zero, liberating the energy consumed in their creation. The amount of energy, liberated in the creation of the Higgs condensate (HQS) is much more than sufficient to create all the particles of the universe.

It also is often alleged that the Higgs field or HQS has no relation with gravity. This can well be true in a static situation. However, likewise superfluids and SC-condensates, the HQS certainly too can locally move. In a superconductor (SC), a constant applied electromotive force (emf) causes an increasing phase gradient along the conductor and flow of the SC-condensate at an increasing 
velocity, proportional to the magnitude of the phase gradient. Once excited, this current is perfectly and perpetually persistent, as demonstrated by numerous experiments. The current can be stopped only by an opposite emf. Applying a magnetic field on the SC, the changing vector potential creates an electric field, causing phase displacements and hence phase disorder, elevating the energy of the SC-condensate. The phase displacements generate screening currents that are associated with a Lorentz force field that usually expel the magnetic field out from the superconductor and thereby lowering the energy of the SC-condensate. The Higgs mechanism [8] [9] is the perfect HQS analog of the Meissner effect. Apparently, the nuclear fields are associated with an HQS analog of the EM vector potential that too causes phase gradients and phase disorder, elevating the energy of the Higgs condensate. The corresponding phase gradients create a screening velocity field of the HQS in the space round the matter bodies. If these screening velocity fields are non-uniform, they refract mutually the matter waves of the interacting particles, generating an inertial dynamics (with conservation of the total linear and angular momenta). This inertial dynamics is, according to Einstein's principle of equivalence, gravitational dynamics.

In his Special Theory of Relativity (STR), Einstein has abolished the idea of an absolute reference and absolute motions. In this scenario, any free (non-accelerated) body, moving in the empty ordinary space under no external force is a proper Lorentz frame, in which stationary clocks show proper time and, with respect to which, the velocity of light is isotropic. In the General Theory of Relativity, Einstein introduced the principle of equivalence, extending locally the view of the STR to free motions within gravitational fields. He assumes that locally non-accelerated (free-falling) bodies (zero external force) too are locally proper references. Accordingly, a free-falling elevator and or orbiting bodies are proper Lorentz frames in which stationary clocks show proper time and the velocity of light is isotropic.

Clocks stationary within gravitational fields are well-known to run slow exactly as predicted by GR. However, actually it is well-known that the gravitational slowing by the solar field is absent on the GPS clocks, moving with earth round the sun [13] [14] [15]. This absence and the well-known absence of light anisotropy with respect to earth both demonstrate that the orbital motion of earth cancels locally the effects of the solar field. From the present HQS dynamics view, these observations can make a sense only if the orbiting earth is stationary with respect to the local HQS, which entails the conclusion that the HQS necessarily is moving round the sun consistently with the earth's orbital motion [16] [17] [18]. Current theories explain the absence of the solar gravitational slowing of the GPS clocks in terms of the principle of equivalence. However, according to this interpretation of the principle of equivalence, the orbital motion of the GPS clocks with the GPS satellites round earth, in exactly the same conditions, too should cancel the gravitational slowing by the earth's field on them. Nevertheless, the gravitational clock slowing by the earth's field is well observed. 
Section 8.B5 solves this problem.

Within gravitational fields, all of Einstein's local free-falling inertial references are mutually non inertial. How can they all be proper references? According to the present work, velocity with respect to the local HQS and not relative velocity is the cause of all the so-called relativistic effects. Therefore, only references, stationary with respect to the local HQS are proper Lorentz frames and only clocks stationary in such proper references can display proper time and moreover only with respect to such proper references is the velocity of light isotropic. Bodies anyhow moving with respect to the local HQS are not proper Lorentz frames. The velocity of light with respect to these non-proper references cannot be isotropic and clocks, stationary in such non-proper references do not show proper time. The next Section solves this stalemate.

\section{The Ingenious Outside-Inside Centrifuge Mechanism of Gravity That Einstein Has Missed}

If the HQS gives inertial mass to the elementary particles, it necessarily is responsible too for the gravitational fields, because it is mass that generates the gravitational fields. It is well-known that clocks stationary within a gravitational field show exactly the gravitational slowing predicted by GR. However, recently the GPS clocks, moving with earth round the sun, have shown that the gravitational slowing by the solar field is absent on them. Visibly, the orbital motion of earth cancels the effects of the solar gravitational field, which runs into conflict with Equation (3).

Current theories explain the absence of the solar gravitational slowing on the GPS clocks by the solar field in terms of the principle of equivalence. Accordingly, in the Lorentz frame of earth, earth is stationary, which is alleged to cancel locally the effects of the solar gravitational field. However, the GPS clocks too are moving with the GPS satellites round earth in exactly the same conditions, which too should cancel the effect of the earth's field. However, the observations show different. The gravitational slowing by the earth's field $\left(1.67 \times 10^{-10} \mathrm{sec} / \mathrm{sec}\right)$ is well present on them. It hence is clear that the absence of the gravitational slowing of the GPS clocks by the solar field, the absence of light anisotropy with respect to the moving earth together with the non-absence of the gravitational slowing by the earth's field on the GPS clocks, these last moving along orbits making 55 degrees with the equator, demonstrate that only orbital motions along direct circular equatorial orbits cancel locally all the effects of the gravitational fields.

In the language of the present work, the Higgs Quantum Space (HQS) materializes the local Lorentz frames ( $L F s)$, turning them into local proper $L F s$, intrinsically stationary with respect to the local HQS. Only LFs, stationary with respect to the local HQS are proper LFs. On moving, the HQS carries the local proper LFs with it. If the motion of the HQS and hence of the local proper LFs through our laboratories, is spatially uniform, it gives rise only to light anisotropy. However, if its motion is non-uniform, besides light anisotropy, it refracts 
the matter waves and light, creating inertial dynamics for the matter particles moving with respect to the local HQS and curving the path of light. According to Einstien's principle of equivalence, this inertial dynamics is gravitational dynamics.

The coming Sections 6, 7 and 8 show that motion of the HQS, round the sun, according to a Keplerian velocity field, consistent with the planetary motions, accurately creates the observed gravitational dynamics and all the observed effects of the gravitational fields on light and on clocks. Please see also References Refs. [16] [17] [18]. In terms of usual spherical coordinates the Keplerian velocity field of the HQS, created by a spherically symmetric source, has the very simple form:

$$
\boldsymbol{V}(r)=(G M / r)^{1 / 2} \boldsymbol{e}_{\phi}
$$

This is a velocity field only along the $\phi$ spherical coordinate round the $Z$ axis $(\theta=0)$, in which the magnitude of the velocity of the HQS is spherically symmetric, $G$ is the gravitational constant, $M$ is the mass of the gravitational source (sun), $r$ is the radial spherical coordinate and $\boldsymbol{e}_{\phi}$ is a unit vector along the azimuthal spherical coordinate $\phi$.

The present work associates the central idea of GR, according to which the gravitational pull is an inertial pull and the central idea of the Higgs theory, according to which the HQS gives mass and governs the inertial motion of matter-energy and replaces Einstein's spacetime curvature by a Keplerian velocity field of the HQS. This Keplerian velocity field is the quintessence of the gravitational fields, in which however the gravitational dynamics is not described in terms of geodesic motions, however in terms of inertial motions (wave propagation) in the Keplerian velocity of the HQS. In this scenario, the gravitational acceleration is a centrifugal acceleration and the gravitational pull is a centrifugal pull, which is a fictitious force. Within this velocity field, the observed gravitational dynamics, the gravitational pull, the absence of the solar gravitational slowing of the GPS clocks by the solar field as well as the absence of the light anisotropy with respect to earth are all genuine and natural outcomes of this Keplerian velocity field of the HQS. These observations are all authentic signatures of the true physical mechanism of gravity in action.

Motion of this HQS according to a Keplerian velocity field naturally and perfectly implements the ingenious outside-inside and inside-outside spherical centrifuge scenario, observed within the gravitational fields. No other imaginable physical mechanism is able to implement this outside-inside centrifuge mechanism. This ingenious centrifuge mechanism is the true hush-hush of the gravitational physics that GR has missed. It also naturally and accurately accounts for all the effects of the gravitational fields on light and on clocks, as will be shown in the coming Sections 6, 7 and 8.

\section{Einstein's Theory of Relativity in the Scenario of the HQS Dynamics}

It is important to note that, in the scenario of the HQS, the velocity of light $c$, in 
the empty space, is fixed and isotropic with respect to the local HQS and not with respect to the reference of every possible observer, as postulated in the theory of relativity. In the scenario of the HQS, the velocity of light in free space (vacuum) is the characteristic velocity at which the HQS propagates the local phase perturbations of the Higgs order parameter. The HQS is ruled by quantum physics. Therefore, the local phase perturbations of the Higgs order parameter and the consequent steady state velocity fields of the HQS (excitations), caused by the elementary particle fields (vector bosons, quarks and leptons), which cause increase of the energy of the HQS, are ruled by quantum physics. The particle fields are confined by the Higgs mechanism down to $10^{-19} \mathrm{~m}$ and quantized according to well defined rules, which lowers the energy of the HQS.

The electromagnetic (EM) field does not couple to the HQS and is not confined by the HQS. Therefore, photons have no rest mass and propagate in empty space at the characteristic propagation velocity $c$, however with respect to the local HQS and not with respect to any observer. The electric field however is not disconnected from the HQS, because the particles, porting the electric charges, couple to the HQS via the hypercharges. Moreover, due to the conservation of electric charge, the number of positive and negative charges necessarily is the same. Therefore, the electric field largely confines itself, so that, from a large scale point of view, only the effects of the gravitational fields are relevant.

Any local reference, stationary with respect to the local HQS, is a local proper Lorentz frame (LF), with respect to which the velocity of light $c$ is fixed and isotropic. However, references moving with respect to the local HQS are non-proper LFs and therefore the (one-way) velocity of light and of neutrinos is anisotropic with respect to them. Within the gravitational fields, the HQS itself is moving according to the Keplerian velocity field Equation (5). In this motion, the HQS carries the local proper LFs with it. Therefore, within the gravitational fields, the local velocity vector of the HQS $\boldsymbol{V}=(G M / r)^{1 / 2} \boldsymbol{e}_{\phi}$ adds up to the fixed velocity vector $\boldsymbol{c}$ of light and the effective velocity of light $c^{\prime}$ is given by $\boldsymbol{c}^{\prime}=\boldsymbol{c}+\boldsymbol{V}$. However, as shown in Section 4, earth is stationary with respect to the local HQS in the solar and the galactic velocity fields and this is why the velocity of light is isotropic with respect to earth and the GPS clocks display proper time.

Clocks are devices that count time in terms of a time standard, which can be a classical or quantum oscillator. The time standard of atomic clocks is the period $T$ of electromagnetic (EM) oscillations in an EM cavity, tuned to the well-defined frequency of the hyperfine transition of Cs atoms. Such clocks measure the time evolution with an incredible precision of a tenth of a nanosecond $\left(\sim 10^{10} \mathrm{sec}\right)$ or even better. Their time standard is of course affected by velocity with respect to the local HQS (not by relative velocity). Within gravitational fields, the HQS is moving round the gravitational sources according to the Keplerian velocity fielded Equation (5). Therefore, clocks, stationary in the ordinary space within a gravitational field, are implicitly moving at a velocity $-(G M / r)^{1 / 2} \boldsymbol{e}_{\phi}$ with respect to the local HQS. This implicit velocity and not the local escape velocity, is 
the cause of the gravitational clock slowing. From the present view, the gravitational clock slowing and the slowing of speeding clocks in free space are due both to the same velocity with respect to the local HQS. This clock slowing however is different for transverse or longitudinal oscillation of the time standard.

From the view of GR, the coefficient of the last term, in Equation (2), is the square of the effective velocity $c^{\prime}$ along the time axis (time evolution), where $c$ and $2 G M / r$ are intrinsically orthogonal. The effective velocity is given by the formula of Pythagoras $c^{\prime}=\left(c^{2}\left(1-2 U / c^{2}\right)\right)^{1 / 2}=\left(c^{2}-2 G M / r\right)^{1 / 2}$, where $2 G M / r$ is the square of the local escape velocity. To first order, the gravitational slowing is $t_{0}(G M / r) / c^{2}$, which is well observed on clocks stationary in the earth's gravitational field. Einstein never has considered the possibility of longitudinal oscillations of the time standard or light round-trips, because, in his view, any ordinary velocity is intrinsically orthogonal to the time axis.

From the viewpoint of the present work, the gravitational time dilation of clocks, stationary within a gravitational field in the ordinary space, is due to the implicit velocity $\left(\boldsymbol{v}_{\text {impl }}(r)=-(G M / r)^{1 / 2} \boldsymbol{e}_{\phi}\right)$. In this scenario, the period $T$ for longitudinal EM oscillations of the clock's time standard is lengthened, given by $\left(T_{l}=T_{0}\left(1-(G M / r) / c^{2}\right)^{-1}\right)$ and to first order the gravitational slowing of the clock is $t_{0}(G M / r) / c^{2}$. However, for transverse oscillations, the gravitational slowing is reduced to one half this value. Moreover, depending on the direction of observation, the dipole EM radiation, emitted by speeding atoms, can be selective for longitudinal or transverse EM oscillations. Dipole photons emitted along the motion of the atoms are selective for transverse EM oscillations.

Considering only transverse oscillations of the time standard, the effective time evolution (velocity along the time axis), in free empty space, is obtained by the formula of Pythagoras $c^{\prime}=\left(c^{2}-v^{2}\right)^{1 / 2}$, where $c$ is the fixed velocity of light with respect to the local HQS in zero gravitational field and $v$ is the ordinary velocity of the clock with respect to stationary HQS. However, within a gravitational field, the HQS is itself moving according to the Keplerian velocity field Equation (5) along $+\phi$. In this case the local effective time evolution (velocity along the time axis), given by a clock, stationary in the ordinary space within the gravitational field too can be calculated by the formula of Pythagoras $c^{\prime}=\left(c^{2}-G M / r\right)^{1 / 2}$, where $c$ is the intrinsic time evolution (velocity along the time axis) of the clock's time standard (Cs atoms) in zero field and where $G M / r$ is the square of the implicit velocity along $-\phi$. For longitudinal oscillations of the clock's time standard, the period is $\left(T=T_{0}\left(1-(G M / r) / c^{2}\right)^{-1}\right)$ and the rate of the clock is $t_{l}=t_{0}\left(1-(G M / r) / c^{2}\right)^{-1}$. To first order, this gives a gravitational clock slowing of $\approx t_{0}(G M / r) / c^{2}$, the same as given by GR.

Now, the first term of the right hand side in Equation (2) describes stretching of the radial distances, due to the spacetime curvature in the gravitational field, given by $\mathrm{d} r^{2}=\mathrm{d} r_{0}^{2} /\left(1-2 U / c^{2}\right)$. According to GR, from the view of an external observer, this stretching causes an apparent reduction of the velocity of light, 
propagating toward the gravitational center. However, from the view of the present HQS dynamics, the velocity of a light beam, propagating toward the gravitational center, decreases really and not apparently. It decreases because it necessarily needs to develop an implicit orthogonal velocity component $(G M / r)^{1 / 2}$ along $-\phi$. The time interval, necessary for light to propagate a distance $\mathrm{d} r$ along the radial coordinate, is $\mathrm{d} t=\mathrm{d} r / c=\mathrm{d} r_{0} /\left(c^{2}-G M / r\right)^{1 / 2}$, where $\left(c^{2}-G M / r\right)^{1 / 2}$ is again the effective velocity $c^{\prime}$ of light, however along the radial coordinate, obtained by the formula of Pythagoras. However this $c^{\prime}$ is only the radial velocity component of light. The other component is $(G M / r)^{1 / 2}$ along $-\phi$. Visibly, GR scales the radial distances in units of the effective radial velocity of light.

\section{The Gravitational Dynamics in the Solar System and on Earth}

The Keplerian velocity field of the HQS (Equation (5)), creating the solar gravitational field is closely consistent with the planetary orbital motions and achieves $436 \mathrm{~km} / \mathrm{sec}$ on the solar surface. This Keplerian velocity field carries with it the planets and the local proper LFs along direct nearly circular equatorial orbits without the need of a central force field. This makes the gravitational dynamics, observed within the solar system, totally obvious. The planetary orbits lie all within the equatorial plane of the solar Keplerian velocity field. So also are practically all planetary satellite orbits. Along such orbits these bodies are all very closely stationary with respect to the local moving HQS (local proper LFs). This motion along direct, circular equatorial orbits is in fact their local resting condition that minimizes their kinetic energy with respect to the local HQS and makes their gravitational (Keplerian velocity) fields very closely spherically symmetric. Excepting only for the very small tides, this cancels locally all the effects of the solar gravitational field on matter, on light and on clocks, exactly as assumed by the current theories with base in the principle of equivalence.

The velocity of light is closely isotropic with respect to earth, not because of the intrinsic isotropy of light, however because earth is stationary with respect to the local moving HQS (local LFs), the medium propagating light. Also clocks, moving with earth round the sun, display very closely proper time, because they too are very closely stationary with respect to the local HQS in the solar Keplerian velocity field. In fact, the small ellipticity of the planetary orbits evidences that their velocity with respect to the local HQS is only of a few hundreds of meters per second. In the case of earth, the effect of this small velocity on light and on clocks is far too small to be detected even by the current most sensitive instruments. However, the refraction rate of this small velocity by the non-uniform velocity in the solar Keplerian velocity field along a whole year is sufficient to make the orbits of the planets a little bit elliptic and to cause the perihelion precession. Please see details in the next Section $\mathbf{8}$.

The velocity of the HQS in the Keplerian velocity field (Equation (5)) round 
earth, in the sense of the Moon's orbital motion (from West to East) and creating the earth's gravitational field, achieves $7.91 \mathrm{~km} / \mathrm{sec}$ on surface. However, as earth rotates only very slowly $(465 \mathrm{~m} / \mathrm{sec}$ at the equator) toward the East, the earth-based laboratories are moving with respect to the local HQS toward the West at an implicit velocity, ranging from $7.445 \mathrm{~km} / \mathrm{sec}$ at the equator up to 1.91 $\mathrm{km} / \mathrm{sec}$ in the Polar Regions. Refraction of this implicit velocity creates the gravitational acceleration and the centrifugal pull toward the earth's center on matter, observed on the earth-surface. It also creates the very small light anisotropy of nearly $8 \mathrm{~km} / \mathrm{sec}$, barely detected by only a few of the most sensitive Michelson light anisotropy experiments [19]. Please see details in Section 8.2. It also gives rise to the very small gravitational slowing of the atomic clocks and the spectral red-shifts, observed on earth. However, although very small, these effects of the earth's field still are much more relevant than the effects by the solar field on earth. The effects of the solar field are extremely small because the orbiting earth is almost stationary with respect to the local HQS in the solar field. These effects simply seem to be absent.

The Keplerian velocity field of the HQS is intermediary between rigid body rotation and potential flow with zero curl. The form of the velocity field of quantum fluids is determined by the limit of non-viscous flow. Due to the order parameter, the differential flow of quantum fluids can be in viscid up to a certain limit of the velocity gradient, even if the curl of the velocity field is non-zero from the classical physics point of view. This property is well-known from the exponential fall of the screening current density (screening velocity fields) in superconductivity. In the HQS this limit determines the $(1 / r)^{1 / 2}$ dependence of the Keplerian velocity field. The curl of the Keplerian velocity field Equation (5) has a form very similar to the magnetic field of a magnetic dipole.

In the Keplerian velocity field, the $\phi$ and $r$ velocity components of a particle with respect to the local HQS are refracted in opposite senses. This characterizes a hyperbolic and not a trigonometric rotation. The differential equation, describing the effects of these refraction rates in the case of a spherically symmetric gravitational source, will be constructed and solved in the coming paragraphs.

In the Keplerian velocity field, the local velocity distribution of the HQS through the $[r, \theta]$ plane seen by a particle with a velocity along the $\phi$ direction $\left(v_{\phi}\right)$ and its de Broglie wave fronts in the $[r, \theta]$ plane, corresponds to rotation of the local HQS and of the local inertial reference (IR) round an overhead axis. The refraction rate corresponds to an instantaneous angular velocity $\boldsymbol{W}_{\phi}(r)$, to be determined, (please see Equation (6)). This rotation axis lies well above the position of the particle. However, the wave-fronts in the $[\theta, \phi]$ plane of the radial velocity component $v_{r}(t)$ do not see this rotation rate round the overhead axis. They see the opposite rotation of the HQS round the gravitational center $\boldsymbol{W}_{r}(r)$. The $\theta$ velocity component is not refracted at all, because the velocity field Equation (5) has no component along $\theta$. Besides these refraction rates, there is need of considering the wavelength stretching/compression, due to 
the deformations of the HQS in the Keplerian velocity field along certain directions. This affects the velocity of the particles according to de Broglie's equation $p=h / \lambda$. However the $r$ and the $\phi$ velocity components are not affected by these wavelength deformations. Hence, describing the effects of the gravitational field, in terms of these velocity components and their effective rotation rates, the effects of wavelength stretching/compression are automatically taken into account.

In order to obtain quantitative values for the refraction rates of the $r$ and $\phi$ velocity components of the effective velocity (with respect to the local HQS), the orbital velocity $v_{o r b}$ of a particle with a very small mass $m$ in the gravitational field of a very large mass $M$, along an elliptic orbit ( $\epsilon=0.5$ ) was computed and plotted very precisely in Figure 1. The values of $v_{o r b}$ together with the Keplerian

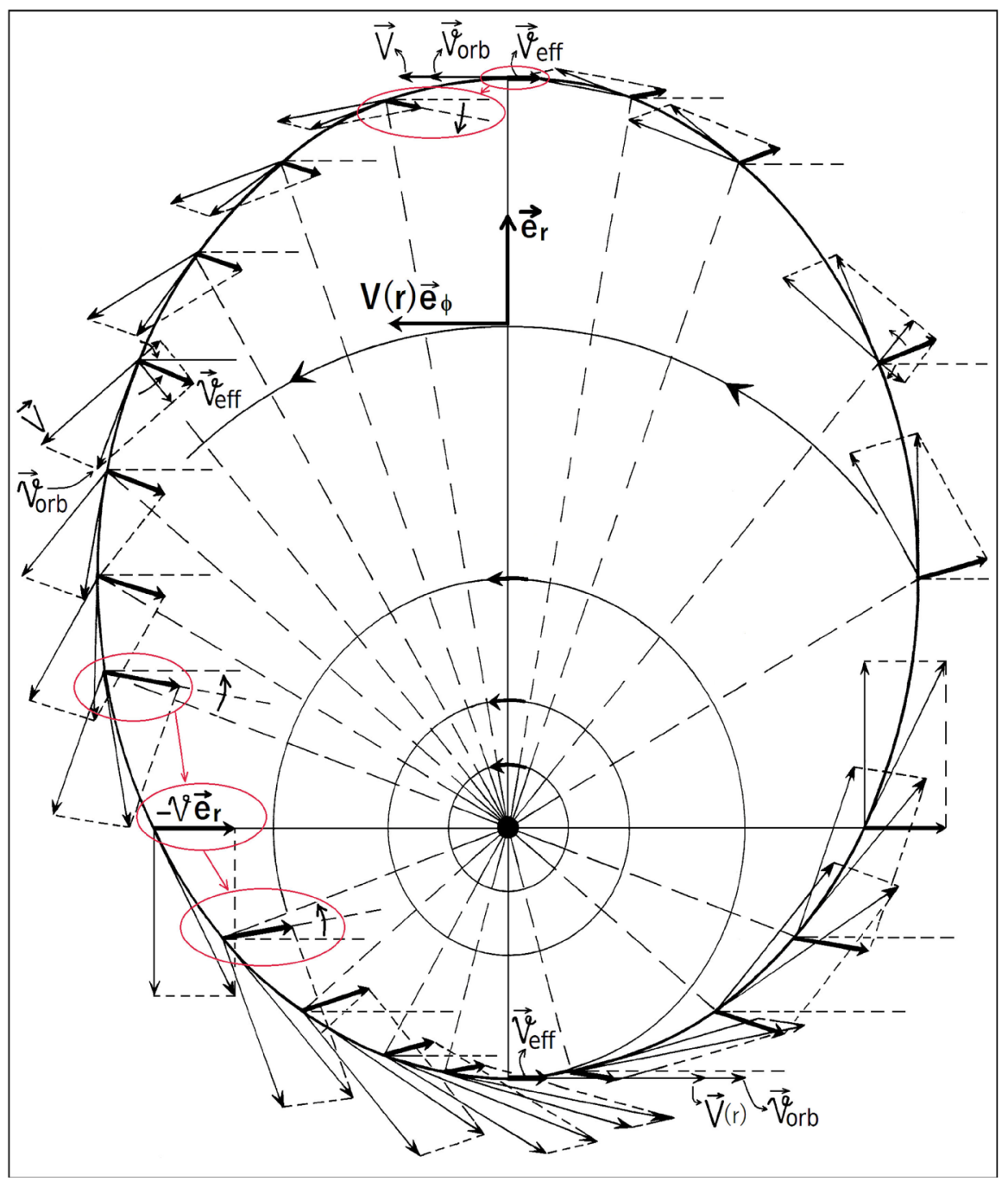

Figure 1. The figure is a very precise graphical representation. The velocity diagrams are shown at a large number of positions along the elliptic orbit, where $v_{\text {eff }}$ is represented by the bulky arrows, pointing nearly toward the right. At the top, the rotation rate of the effective (implicit) $-\phi$ velocity component can be read. The rotation rate of the $-r$ velocity component can be read at the left. Please see the encircled diagrams. 
velocity $V(r)$, given by Equation (5) and the effective velocity $v_{\text {eff }}$, determined by the equation $v_{\text {eff }}=\boldsymbol{V}(r)+\boldsymbol{v}_{\text {orb }}$ are plotted at a large number of points along the orbit. Please see carefully especially the encircled velocity diagrams. The obtained refraction rates of the $-r,-\phi$ and the $\pm \theta$ velocity components are:

$$
\begin{gathered}
\boldsymbol{W}_{r}(r)=-\frac{1}{2}\left[G M / r^{3}\right]^{1 / 2} \boldsymbol{e}_{\theta} \\
\boldsymbol{W}_{\phi}(r)=+\left[G M / r^{3}\right]^{1 / 2} \boldsymbol{e}_{\theta} \\
\boldsymbol{W}_{\theta}(r)=0
\end{gathered}
$$

Please note the opposite sighns in the rotation rates of the $r$ and the $\phi$ velocity components.

The implicit velocity along $-\phi$ of the small particle of mass $m$ stationary in the gravitational field, of a very much larger mass $M$ is given by:

$$
\boldsymbol{V}_{\text {impl }}=-(G M / r)^{1 / 2} \boldsymbol{e}_{\phi}
$$

The refraction rate of this implicit velocity, according to Equation (6b), generates an instantaneous ordinary vertical downward acceleration given by:

$$
\boldsymbol{g}(r)=W_{\phi}(r) \boldsymbol{e}_{\theta} \times V_{\text {impl }}(r)\left(-\boldsymbol{e}_{\phi}\right)=-G M / r^{2} \boldsymbol{e}_{r}
$$

Equation (8) has exactly the form of a formal expression for centrifugal accelerations in a rotating reference and $\boldsymbol{g}(r)$ has clearly the nature of a centrifugal acceleration. It is a spherically symmetric field of centrifugal accelerations (by fictitious forces) toward the gravitational center.

A body hold fixed with respect to the ordinary space coordinates within the Keplerian velocity field Equation (5), in fact will be stationary with respect to a non-inertial reference that is implicitly rotating oppositely to the local (true) inertial reference, this last truly rotating in the ordinary space according to Equation (6b). Bodies, stationary within the non-inertial earth-based laboratories, necessarily are implicitly moving along circular paths within the local true rotating IR. This however they can do only under a real upward centripetal force. This implicit motion along a circular path is not free motion and also does not correspond to geodesic motion in the curved spacetime of GR. While according to GR a free-falling elevator is locally a proper inertial reference, according to the HQS dynamics it is moving along $\phi$ at a velocity $-(G M / r)^{1 / 2}$ with respect to the local HQS and hence cannot be a proper reference and also not a proper Lorentz frame. Clocks in such free-falling references are predicted to run slow and the velocity of light is predicted to be anisotropic.

Equation (8) is sufficiently precise only for free-fall along short distances. For large distances of free-fall of a small mass $m$ in the field of a very large mass $M$, the following elementary linear differential equation must be solved:

$$
\frac{\mathrm{d} \boldsymbol{v}(r(t))}{\mathrm{d} t}=A \boldsymbol{v}_{0}
$$


where $\boldsymbol{v}$, is the column matrix of the (ordinary) $r$ and the (implicit) $\phi$ velocity components of the effective velocity with respect to the local HQS:

$$
\boldsymbol{v}(t)=\left(\begin{array}{l}
v_{r}(t) \\
v_{\phi}(t)
\end{array}\right)
$$

In Equation (9), $\boldsymbol{A}$ is the hyperbolic rotation matrix, defined in terms of the rotation rates, given in Equation (6):

$$
\boldsymbol{A}=\left(\begin{array}{cc}
0 & W_{\phi} \\
-W_{r} & 0
\end{array}\right)=\left(\begin{array}{cc}
0 & W \\
\frac{1}{u} W & 0
\end{array}\right)
$$

where $W=G M / r^{3}$ and $u=2 M /(M+m)$, the value of which varies from 1 , for $m=M$, up to 2 for $m \ll M$.

Dividing both sides of Equation (9) by $\boldsymbol{v}_{0}$, multiplying them by $\mathrm{d} t$ and integrating the left hand side from $v_{0}$ to $v$, develops into:

$$
\log \frac{\boldsymbol{v}(t)}{\boldsymbol{v}_{0}}=\int_{0}^{t} A\left(r\left(t^{\prime}\right)\right) \mathrm{d} t^{\prime}
$$

where $A d t$ is an infinitesimal rotation round parallel axes. Equation (12) can be re-written in the exponential form as:

$$
\boldsymbol{v}(t)=\exp \left[\int_{0}^{t} A\left(r\left(t^{\prime}\right)\right) \mathrm{d} t^{\prime}\right] \boldsymbol{v}_{0}
$$

Expanding the exponential in series and adding up the terms of the series from $n=0$ to $n=\infty$ results in:

$$
\begin{aligned}
v(t) & =\sum_{n=0}^{\infty} \frac{1}{n !}\left(\begin{array}{cc}
0 & \Theta(t) \\
\frac{\Theta(t)}{u} & 0
\end{array}\right)^{n}\left(\begin{array}{l}
v_{r}(0) \\
v_{\phi}(0)
\end{array}\right) \\
& =\left(\begin{array}{cc}
\cosh \left(\frac{\Theta(t)}{\sqrt{u}}\right) & \sqrt{u} \sinh \left(\frac{\Theta(t)}{\sqrt{u}}\right) \\
\frac{1}{\sqrt{u}} \sinh \left(\frac{\Theta(t)}{\sqrt{u}}\right) & \cosh \left(\frac{\Theta(t)}{\sqrt{u}}\right)
\end{array}\right) \times\left(\begin{array}{l}
v_{r}(0) \\
v_{\phi}(0)
\end{array}\right)
\end{aligned}
$$

The value of $\Theta(t)$ can be computed by integration:

$$
\begin{aligned}
\Theta(t) & =\int_{0}^{t} W\left[r\left(t^{\prime}\right)\right] \mathrm{d} t^{\prime} \\
& =\int_{r_{0}}^{r^{\mathrm{CM}}}\left[\frac{G M}{\left(r^{\mathrm{CM}}+R^{\mathrm{CM}}\right)^{3}}\right]^{1 / 2} \frac{\mathrm{d} r^{\mathrm{CM}}}{\dot{r}^{\mathrm{CM}}} \\
& =-\sqrt{u} \cosh ^{-1}\left[\frac{r_{0}^{\mathrm{CM}}}{r^{\mathrm{CM}}(t)}\right]^{1 / 2}
\end{aligned}
$$

Inversion of the final term of Equation (15) results in expressions for the hyperbolic cosines and sines:

$$
\cosh \left(\frac{\Theta(t)}{\sqrt{u}}\right)=\sqrt{\frac{r_{0}^{\mathrm{CM}}}{r^{\mathrm{CM}}}}=\sqrt{\frac{r_{0}}{r}}
$$




$$
\sinh \left(\frac{\Theta(t)}{\sqrt{u}}\right)=\sqrt{\frac{r_{0}^{\mathrm{CM}}-r^{\mathrm{CM}}}{r^{\mathrm{CM}}}}=\sqrt{\frac{r_{0}-r}{r}}
$$

where the last equality is obvious. Using this result and noting that $v C M_{\phi}=v_{\phi}$, Equation (14) becomes:

$$
\left(\begin{array}{l}
v_{r}(t) \\
v_{\phi}(t)
\end{array}\right)=\left(\begin{array}{l}
\sqrt{r_{0} / r} \sqrt{u\left(r_{0}-r\right) / r} \\
\sqrt{\left(r_{0}-r\right) / u r} \sqrt{r_{0} / r}
\end{array}\right) \times\left(\begin{array}{l}
v_{r}(0) \\
v_{\phi}(0)
\end{array}\right)
$$

The particular solution of Equation (9), for free fall of $m$ in the field of $M$ $(m \ll M)$ ), on from $r_{0}$ and initial rest, where $v_{r}(t=0)=0$ and $\boldsymbol{v}_{\phi}(0)=\boldsymbol{V}_{\text {impl }}\left(r_{0}\right)=-\left(G M / r_{0}\right)^{1 / 2} \boldsymbol{e}_{\phi}$, the final solution of Equation (9) is:

$$
\begin{gathered}
v_{r}(t) \approx-\left[2\left(\frac{G M}{r(t)}-\frac{G M}{r_{0}}\right)\right]^{1 / 2} \boldsymbol{e}_{r} \\
v_{\phi}(t)=-V_{\text {impl }}\left(r_{0}\right)\left[\frac{r_{0}}{r}\right]^{1 / 2}=-\left[\frac{G M}{r(t)}\right]^{1 / 2} \boldsymbol{e}_{\phi}
\end{gathered}
$$

Equation (18a) is just the well known expression for the observed vertical free-fall velocity on from rest at $r_{0}$, directly showing that the kinetic energy is equal to the difference between the final and the initial potential energies. Equation (18b) is just the implicit (imaginary) velocity as a function of the radial position $r$. This shows that the refraction rate of the radial velocity component just compensates for the increase of the velocity field as a function of the decrease of the radial coordinate. This assures that free-fall of the particle, on from rest, goes along a vertical (radial) path and hence assures conservation of the angular momentum about the gravitational center. Please observe that, for free-fall on from infinity $\left(r_{0}=\infty\right)$, the vertical velocity $v_{r}(r(t))$ is exactly $\sqrt{2}$ times larger than the $\phi$ velocity, which arises directly from Equation (18) and accomplishes the Virial theorem.

\section{Symmetry of the HQS Dynamics Gravitation with Orbital Motions}

Consider free-fall experiments at the equatorial region of a rotating planet of radius $R$ like earth rotating at an angular velocity $\omega$ in a direct or retrograde sense round the same axis as the Keplerian velocity field Equation (5). The effective velocity $\boldsymbol{v}_{\text {eff }}$ of such a particle, initially stationary near to the surface of a planet, will be given by:

$$
\begin{aligned}
\boldsymbol{v}_{\text {eff }}(\theta) & =\boldsymbol{V}_{\text {impl }}(R)+\boldsymbol{v}_{\text {rot }}(\theta) \\
& =-\left[(G M / R)^{1 / 2} \mp \omega R \sin \theta\right] \boldsymbol{e}_{\phi}
\end{aligned}
$$

where $\boldsymbol{v}_{\text {rot }}(\theta)=\omega R \sin (\theta)$ is the ordinary velocity, due to the planet's rotation that depends on the latitude via $\sin \theta$. The upper and lower signs are respectively for direct and or retrograde rotation of the planet.

However, rotation of the planet in the same sense as the Keplerian velocity 
field of the HQS, gives rise to a trigonometric rotation rate of the effective velocity $\boldsymbol{v}_{\text {eff }}$ of the particle, due to the planet's rotation that adds up to the refraction rate, given by Equation (6b) and subtracts for retrograde rotation of the planet. The effective rotation rate of the effective velocity vector is:

$$
\boldsymbol{\omega}_{e f f}(\theta)=\frac{1}{R}\left[V_{\text {impl }} \pm\left(v_{r o t}\right)\right] \boldsymbol{e}_{\theta}=\left[\left(G M / R^{3}\right)^{1 / 2} \pm \omega \sin \theta\right] \boldsymbol{e}_{\theta}(20)
$$

where the same convention for the upper and the lower signs as in Equation (19) is used.

Considering the effective velocity (Equation (19) and the effective rotation rate (Equation (20), the effective gravitational acceleration on the planet's surface is:

$$
\boldsymbol{g}_{\text {eff }}(\theta)=\boldsymbol{\omega}_{\text {eff }} \times \boldsymbol{v}_{\text {eff }}=-\left[G M / R^{2}-\omega^{2} R \sin ^{2} \theta\right] \boldsymbol{e}_{r}
$$

The first term in the right hand side of Equation (21) describes the gravitational acceleration toward the gravitational center of the planet in the static situation (see Equation (8)), while the second term is an outward centrifugal term. Most importantly, Equation (21) shows that the effective gravitational acceleration $\boldsymbol{g}_{\text {eff }}(\theta)$ in the rotating planet's Keplerian velocity field is perfectly symmetric for direct or retrograde rotation and thus also for direct or retrograde orbital motion.

For a body in a strictly circular polar orbit with orbital radius $r>R, \boldsymbol{v}_{\text {eff }}$ has velocity components along $-\phi$ as well as along $\pm \theta$. The velocity along $-\phi$ is:

$$
\boldsymbol{v}_{\phi}=\boldsymbol{V}_{i m p}(r)=-[G M / r]^{1 / 2} \boldsymbol{e}_{\phi}
$$

Along theta the velocity is:

$$
\boldsymbol{v}_{\theta}= \pm[G M / r]^{1 / 2} \boldsymbol{e}_{\theta}
$$

While $\boldsymbol{v}_{\phi}$ generates the gravitational acceleration $\boldsymbol{g}(r)=-G M / r^{2} \boldsymbol{e}_{r}$, see Equation (8), the $\theta$ velocity component is not affected directly by the HQS-dynamics, because the velocity field Equation (5) has no velocity component along theta. However, the rotation rate of the $\phi$ velocity component plays the role of a centripetal force accelerating the body toward the gravitational center, bending the $\theta$ velocity toward the earth's surface. This in particular literally implements the idea of Newtonian gravitation. The effective gravitational acceleration for circular polar orbits is:

$$
\boldsymbol{g}_{\text {eff }}(r)=-\left[G M / r^{2}-v_{\theta}^{2} / r\right] \boldsymbol{e}_{r}
$$

where again the first term in the right hand side is the acceleration toward the gravitational center (please see Equation (8)), while the second term is the corresponding usual upward centrifugal effect.

Together the results, expressed by Equations (21) and (24), show that the effects of the HQS dynamics and the effects of the ordinary motion within the gravitational field are completely independent. They are orthogonal. While the 
Keplerian velocity field of the HQS (Equation (5) simulates a central field of fictitious Newtonian gravitational forces, the ordinary orbital motions generate the centrifugal effects, exactly as conceived in Newtonian gravity. This shows that treating the motions, within a spherically symmetric gravitational field, as motions in a hypothetical inertial reference, extending over the whole region, under a hypothetical central field of fictitious gravitational forces although not corresponding to reality, gives closely the observed gravitational dynamics. This explains why the Newtonian gravitational theory, although based in the fictitious gravitational forces, works so well.

\section{The Effects of the Keplerian Velocity Field of the HQS on Light and on Clocks}

\subsection{Effects in the Solar System}

\subsubsection{The Excess Time Delay of Radar Signals in Round-Trips within the Solar System}

According to GR, massive astronomical bodies curve spacetime in their neighborhood, as given for weak fields by Equation (3) in the Introduction. This spacetime curvature bends light signals and lengthens the go-return round-trip path of electromagnetic (EM) signals passing by massive bodies, thereby giving rise to an excess time delay (Shapiro Effect). According to the present work the excess time delay is not caused by increase of the geometrical distances. It is due to the effective velocity of the signals in the Keplerian velocity field of the sun. In the case of round-trips between earth and Venus, the solar velocity field is favorable during the prograde travel, increasing the effective signal velocity and reducing the travel time. It however is unfavorable during the retrograde travels, decreasing the effective signal velocity and increasing the return travel time. The problem is totally analogous to that of light round-trips between two mirrors within a laboratory, moving with respect to the local HQS. However, in the solar Keplerian velocity field, the calculation is a little bit more complicated, because the velocity of the signal depends on position.

In the present work, the excess time delay was numerically calculated along straight line paths, separately for travels from earth to Venus and back from Venus to earth in terms of the effective velocity $v_{\text {eff }}=\boldsymbol{c}_{\mathrm{HQS}}+\boldsymbol{V}$, where $\boldsymbol{c}_{\mathrm{HQS}}$ is the fixed velocity of light with respect to the local moving HQS. This calculation was repeated for a series of paths, having minimum distances from the center of the sun $R=2,4,8,25,50$ and 100 million km respectively. The calculations were made for these round-trips before and after superior conjunction. The excess time delays were calculated dividing the straight line paths into about 420 segments, shorter segments near to the sun, separately for transverse and longitudinal components of the solar Keplerian velocity field along the signal paths and finally integrated over the full path. More than $99 \%$ of the excess time-loss in the retrograde journeys or time-gain in the prograde journeys comes from the longitudinal component of the solar velocity field along the path. The different Earth-Venus 
distances, due to the orbital positions as well as the slightly different signal path, due to the motion of earth during each signal round-trip, was taken into account. The obtained results are given in Table 1 and plotted in Figure 2.

Table 1. The first column in Table 1 gives the values of the nominal impact parameters $R$, the second and third columns give respectively the gain of time (negative) and the loss of time (positive), due to the solar Keplerian velocity field of the HQS in milliseconds (ms) before and after superior conjunction. The forth column gives the effective excess time-delays for full go-return round-trips in microseconds $(\mu s)$.

\begin{tabular}{cccc}
\hline$R\left(10^{6} \mathrm{~km}\right.$ units $)$ & go: $\Delta t(\mathrm{~ms})$ & return: $\Delta t(\mathrm{~ms})$ & $\Delta t_{\text {eff }}(\mu \mathrm{s})$ \\
\hline 100 & -51.987 & 51.999 & 12 \\
50 & -76.697 & 76.725 & 28 \\
25 & -70.007 & 70.056 & 49 \\
8 & -48.585 & 48.679 & 94 \\
4 & -36.699 & 36.833 & 134 \\
2 & -27.165 & 27.351 & 186 \\
-2 & 27.351 & -27.165 & 186 \\
-4 & 36.833 & -36.699 & 134 \\
-8 & 48.679 & -48.585 & 94 \\
-25 & 70.056 & -70.007 & 49 \\
-50 & 76.725 & -76.697 & 28 \\
-100 & 51.999 & -51.987 & 12 \\
\hline
\end{tabular}

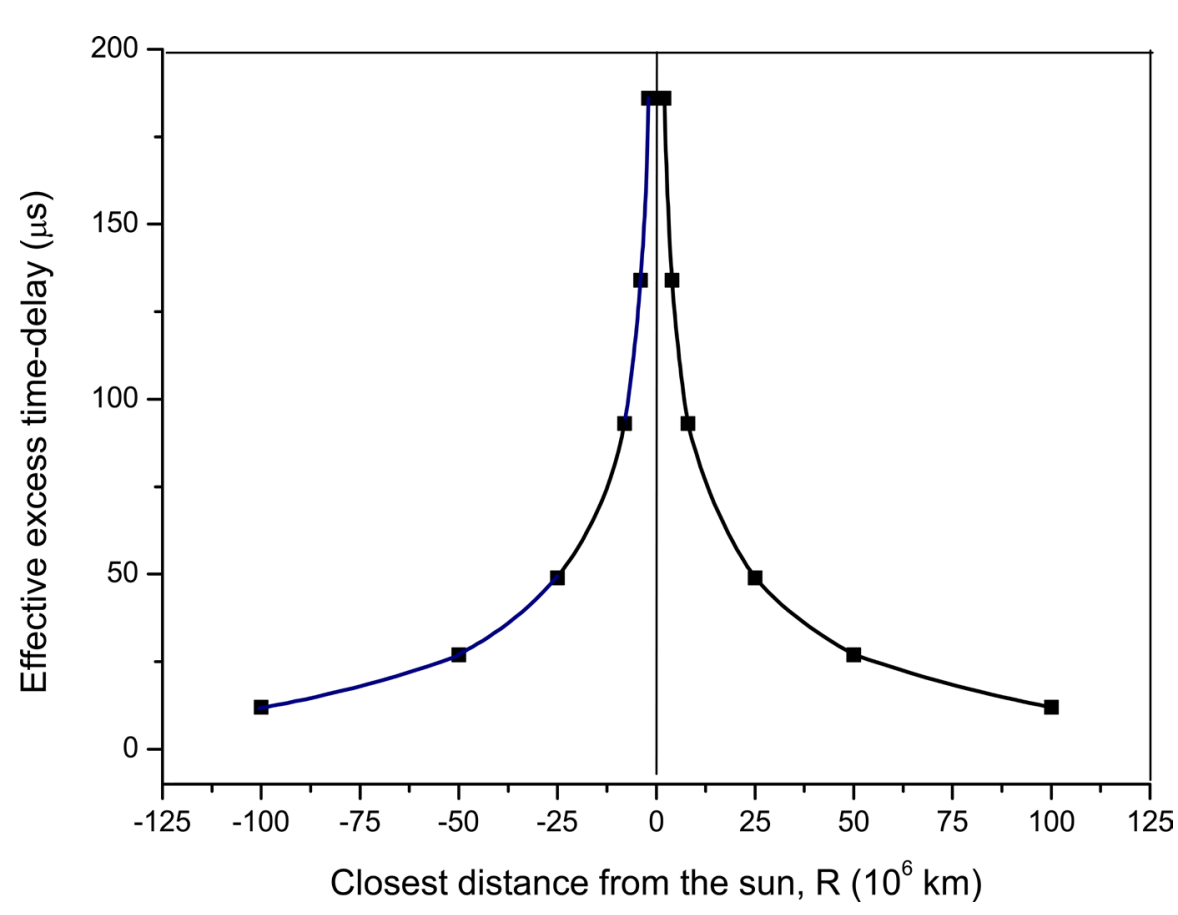

Figure 2. The calculated effective excess time delay, given in microseconds ( $\mu \mathrm{s}$ ), (fourth column in Table 1) for complete go-return round trips. The calculated data curves practically coincide with those, obtained by Shapiro ref. [20]. 
The fact that the Keplerian velocity field of the HQS round the sun correctly produces the effective excess time delay (Shapiro effect) fully corroborates the present HQS-dynamics gravitational mechanism.

\subsubsection{The Gravitational Light Lensing Effect}

Consider two light beams, coming from two distant stars and propagating toward the sun along two parallel paths as shown in Figure 3. First, in the region where the velocity of light $c$ has almost only a radial component, the wave vectors of the two beams will be refracted according to Equation (6a) by a total angle $+\alpha$, thereby the light beams gaining a small velocity component $V(r)=-(G M / r)^{1 / 2}$ along $-\phi$. This component cancels the drag by the solar velocity field. This however reduces the radial velocity component $c_{r}$ to $c_{r}=\left(c_{\mathrm{HQS}}^{2}-V^{2}\right)^{1 / 2}$. Near to the sun, where the solar velocity field achieves 436 $\mathrm{km} / \mathrm{sec}$ and is mostly parallel (left in Figure 3 ) and anti-parallel (at the right) to the beams, refraction by Equation (6b) is dominant and the wave vectors are refracted oppositely by a total angle $-2 \alpha$. Finally, after having passed by the sun and going away in the opposite side, the wave vectors again are refracted according to Equation (6a) by nearly an angle $+\alpha$. However, near to the sun, the solar velocity field is favorable to the prograde ray (left) so that it spends less time near to the sun and hence is refracted by a smaller angle $-(2 \alpha-\delta)$. To the retrograde ray however (right) the solar velocity field is unfavorable and hence it spends a longer time near to the sun and refracts by a larger angle $-(2 \alpha+\delta)$. This differentiated refractions by $\delta$, due to Equation (6b) causes a (symmetric) convergence of the rays that is responsible for the gravitational light lensing effect.

The value of $\delta$ can be calculated simply by multiplying the refraction rate Equation (6b) times the excess (or shortage) of time delays $\Delta t$, listed in Table 1.

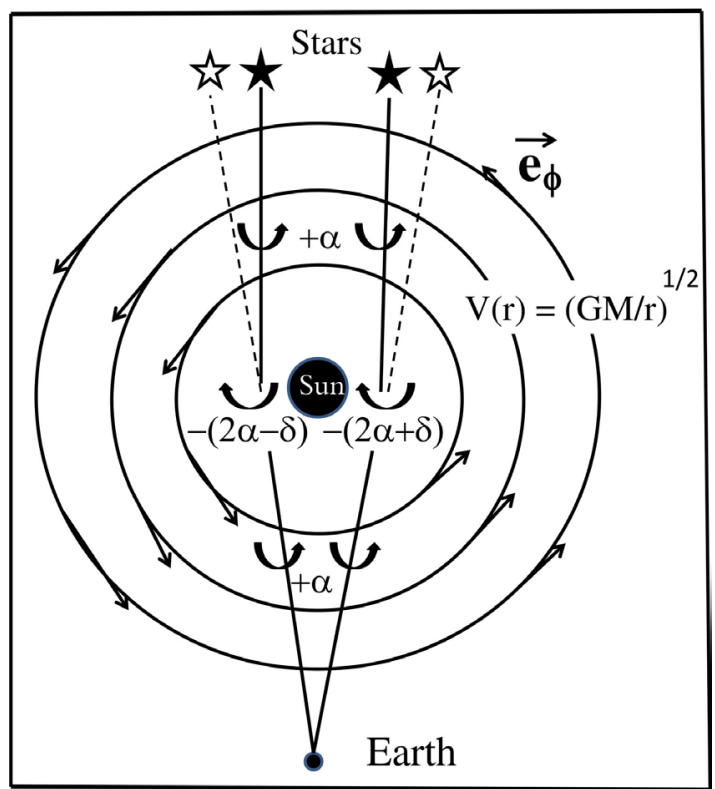

Figure 3. Gravitational light-lensing effect, by a heavy mass (Sun). 
On the solar surface $R=6.9565 \times 10^{8} \mathrm{~m}$ and the refraction rate, given by Equation (6b), is $W_{\phi} \sim 0.0359 \mathrm{deg} / \mathrm{sec}$. Interpolating the value of $\Delta t$ in Table 1 for light passing by the surface of the sun, we find the excess time delay at the retrograde side or the shortage of time at the prograde side of closely $\Delta t= \pm 13.5 \mathrm{~ms}$. The product $W_{\phi} \times \Delta t$ gives $\delta=1.745$ arcsec, which is very closely the observed deflection angle, causing the convergence of light passing close by the solar surface.

\subsubsection{The Non-Synchronous Arrival of the Pulsar Signals to Equidistant Earth-Based Antennas along the Earth's Orbital Motion}

Imaging astronomical objects with the help of interferometric methods can improve the images by orders of magnitude. To this end, it is necessary that the signals from the different receivers be synchronous, which is achieved with the help of the GPS clocks. However, on testing the, so synchronized clocks, by the arrival of the millisecond pulsar signals, there was a surprise. While the arrival of the pulsar signals to the antenna arrays along directions transverse to the earth's orbital motion was synchronous, along the orbital motion of earth the arrival was out of synchrony, up to $4.2 \mu \mathrm{sec}$ refs. [13] [14].

The aberration of star light is an old and well known feature in astronomical observations from earth. Usually the aberration angle is explained in terms of the orbital velocity of earth $\sin \beta=V / c$. However, according to the present work, this aberration contains an additional feature that arises from the refraction rate Equation (6a) of light propagating from distant objects toward the sun. This refraction rate creates a velocity component of the light signal toward $-\phi$ and thereby reduces the radial velocity component to $c_{r}=\left(c_{\mathrm{HQS}}^{2}-V^{2}\right)^{1 / 2}$. Correspondingly it slants the wave fronts by and angle $\beta$, so that the wave fronts reach first the leading part of earth and only $4.2 \mu$ sec later the rear part.

\subsubsection{The Perihelion Precession}

An analogous differentiated refraction rate of the propagation velocity $v$ of an orbiting body as in the preceding Section 8.A3 must be responsible for the perihelion precession of elliptical orbits. At the perihelion $\boldsymbol{v}_{\text {eff }}$ is parallel to the velocity field (prograde) and to the orbital velocity, which displaces it more rapidly (than the HQS), so that it has not time enough to recover the tangential direction. It recovers it only somewhat beyond the ideal perihelion point. In this way the perihelion advances a little bit in the prograde sense in each orbital round-trip.

\subsubsection{Absence of Effects of the Solar Gravitational Field on the GPS} Clocks

In the view of the present HQS-dynamics gravitational mechanism, the slowing of clock rates is caused by velocity with respect to the local HQS and not by relative velocity. Hence, clocks, stationary with respect to the local moving HQS, do not run slow. They show proper time as confirmed by the GPS clocks. The effective velocity of the GPS clocks, moving with earth round the sun, is zero with respect to the local moving HQS because earth is commoving with the local HQS 
in the Keplerian velocity field, creating the solar gravitational field. The effective velocity is given by:

$$
\boldsymbol{v}_{\text {eff }}=\boldsymbol{V}(r)-\boldsymbol{v}_{\text {orb }} \cong(G M / r)^{1 / 2} \boldsymbol{e}_{\phi}-(G M / r)^{1 / 2} \boldsymbol{e}_{\phi} \approx 0
$$

All clocks orbiting in circular equatorial orbits round an astronomical body, which normally is moving itself in a direct circular equatorial orbit round a larger body (star or galaxy), are stationary with respect to the local HQS (local LFs). Such clocks all are naturally synchronous with respect to each other and all show closely the same proper time throughout the universe.

\subsection{Effects of the Earth's Field}

\subsubsection{The One-Way Light Velocity between Artificial Satellites}

In order to measure the one-way velocity of light, it is necessary to have well synchronized clocks at each end of the one-way travel distance. Actually the atomic clocks in orbit can be synchronized by Einstein's method down to $0.1 \mathrm{~ns}$ (time for light to propagate $3 \mathrm{~cm}$ ). This synchronization is especially favorable for clocks along polar orbits. The most precise measurement of the one-way velocity of light was achieved with the help of atomic clocks in the robotic twin satellites of the GRACE project, moving both in the same polar orbit at $500 \mathrm{~km}$ of altitude and separated by $\sim 200 \mathrm{~km}$. In order to measure microgravity effects, these clocks need to be synchronized always to better than $0.16 \mathrm{~ns}$. Continuous exchange of EM signals between these satellites, in both senses, has revealed a clear anisotropy of the signal velocity of nearly $8 \mathrm{~km} / \mathrm{sec}$, backward to the motion of the satellites the same velocity as the orbital velocity of the satellites. [15] This observation evidences that a spatial medium (HQS) propagating the EM signals (light) exists and that it has no relevant velocity along polar orbits. The immediate consequence of this observation is the need of finding out a new interpretation for all the light anisotropy experiments performed in the past century. The next Section 8.B2 implements this.

\subsubsection{The Michelson Light Anisotropy Experiments}

The large majority of the Michelson experiments, searched for light anisotropy due to the orbital and cosmic motion of earth. They all found closely null results. This confirms that the orbiting earth is very closely stationary with respect to the local HQS as also evidenced by the absence of the gravitational slowing of the GPS clocks by the solar field. These null results also show that the solar system is stationary in the velocity field of the HQS, generating the gravitational dynamics of the Milky-Way galaxy and that our galaxy is stationary with respect to the HQS, despite the accelerated expansion of the universe.

From the view of the HQS dynamics, the only motion, that causes relevant anisotropy of the light velocity with respect to the earth-based laboratories, is the local velocity field of the HQS round earth itself in the sense of the Moon's orbital motion and creating the earth's gravitational field. On the earth's surface this velocity reaches $7.91 \mathrm{~km} / \mathrm{sec}$. This predicts light anisotropy on earth of 
somewhat less than $8 \mathrm{~km} / \mathrm{sec}$, a second order effect of only $10^{-10}$, constant the whole day and the whole year and therefore extremely difficult to be detected. Several light anisotropy experiments using laser cavities obtained null results. The small laser cavities are intrinsically unable to detect such low anisotropies, due to their quantized modes. Only a few of the Michelson light anisotropy experiments with the highest sensitivity and rotating within the earth-based laboratories have detected such small anisotropies, constant the whole day and the whole year. Figure 4 displays Miller's results. [19]

\subsection{Gravitational Time Dilation and Gravitational Spectral Red Shifts}

The gravitational slowing of atomic clocks stationary in the earth's gravitational field and the gravitational spectral red-shifts of atoms are now well confirmed experimentally refs. [3] [21] [22]. To first order, these effects are proportional to $(G M / r) / c^{2}$. On the earth-surface their effect is extremely small, in the order of $10^{-10}$ and, excepting by Massbauer resonance scattering, are very difficult to be detected.

\subsubsection{Gravitational Slowing Of Clocks in Direct Circular Equatorial Orbits Round Earth}

According to the present work, slowing of the clock rates is caused by velocity with respect to the local HQS (LFs) and not by relative velocity. The gravitational slowing of the GPS clocks by the solar field, predicted by GR, is absent. These clocks display proper time, because they are stationary with respect to the local moving HQS in the Keplerian velocity field, creating the solar gravitational field. Analogously, clocks, moving in direct circular equatorial orbits round earth, too are stationary with respect to the local HQS in the Keplerian velocity field, creating the earth's gravitational field and too must display proper time. The orbital velocity of the geosynchronous satellites in direct circular equatorial orbits round earth, with an orbital radius of $42,224 \mathrm{~km}$ move at nearly $3.1 \mathrm{~km} / \mathrm{sec}$. Clocks in these satellites are stationary with respect to the local HQS and hence show proper time. Unhappily, to present date such an experiment is not known.

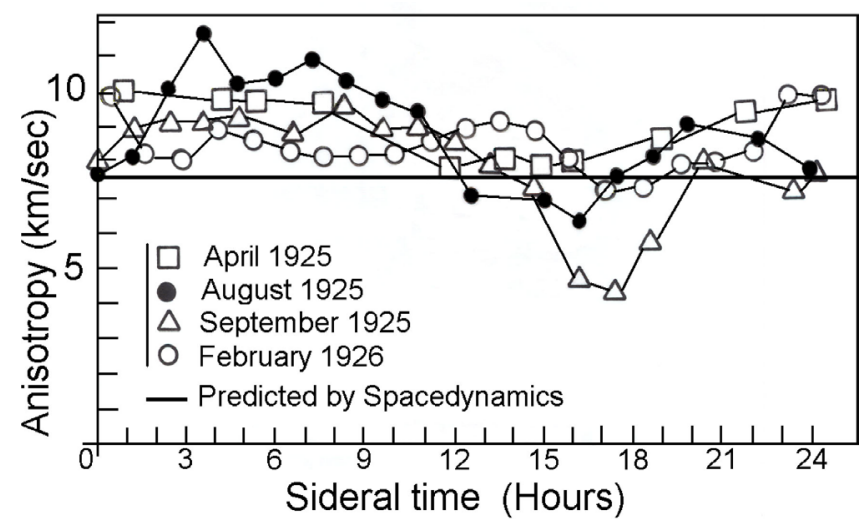

Figure 4. The Nearly West-East light anisotropy, with respect to the earth-based laboratories, obtained 1925 and 1926 by D. Miller that is nearly constant the whole day and the whole year. 


\subsubsection{Effects of the Velocity Field of the HQS on Clocks Moving in Non-Equatorial Circular Orbits Round Earth}

In the view of the present work, clocks in direct circular equatorial orbits show proper time. However, clocks in circular polar orbits round earth are slowed two times more than a clocks stationary at the same altitude and clocks in retrograde circular equatorial orbits are slowed four time more than a clock stationary at the same altitude. In particular the GPS satellites move at $2.02 \times 10^{4} \mathrm{~km}$ of altitude, along non-equatorial circular orbits inclined 55 degrees with respect to the earth's equator and hence have a considerable velocity with respect to the local HQS in the Keplerian velocity field, creating the earth's gravitational field. The GPS clocks have velocity components, given by $v_{0}(1-\cos \alpha)$ along $-\phi$ and $v_{0} \sin \alpha$ along $\pm \theta$, where $v_{0}=3.87 \mathrm{~km} / \mathrm{sec}$ and $\alpha$ is the angle of the orbital velocity $v_{0}$ with respect to the equator or parallels. The effective velocity at the equator is $v_{0}[2(1-\cos \alpha)]^{1 / 2}=3.574$ and the estimated average velocity of the GPS satellites with respect to the local HQS over the entire orbit is $\sim 0.8 \times 3.574=2.86 \mathrm{~km} / \mathrm{sec}$. Analogously the Westward velocity of the earth-based station at Colorado highs with respect to the local HQS is about $7.4 \mathrm{~km} / \mathrm{sec}$. In addition, the velocity of the Cs atoms of $0.255 \mathrm{~km} / \mathrm{sec}$ within the atomic clocks as well as a small transverse Doppler shift, due to the implicit velocity of the earth-based stations with respect to the local HQS must be considered. Altogether these effects achieve closely the observed $4.5 \times 10^{-10} \mathrm{sec} / \mathrm{sec}$. ref. [3].

\subsubsection{Drag of Neutrinos and Light toward the East by the Earth's Keplerian Velocity Field}

From the HQS-dynamics view, the velocity of light in the vacuum has a fixed value ( $c=299792458 \mathrm{~m} / \mathrm{sec}$ ) with respect to the local HQS and not with respect to all the possible inertial references. The velocity of neutrinos is almost equal to the light velocity. Light and neutrinos are dragged, within gravitational fields, by the respective Keplerian velocity fields of the HQS. In the earth's Keplerian velocity field Equation (5), the velocity of the HQS moves from West to East and achieves $V=7.91 \mathrm{~km} / \mathrm{sec}$ on surface. This velocity adds up to the velocity of light and of the neutrinos, giving an effective velocity, given by $c^{\prime}=\boldsymbol{c}+\boldsymbol{V}$, turning their velocity with respect to earth-based laboratories anisotropic. This is a fundamental prediction of the HQS dynamics gravitational mechanism.

In the neutrino experiment from CERN-Geneva-CH to OPERA-Gran Sasso-IT, the neutrinos speeded under-ground along a straight path of $730 \mathrm{~km}$, making 58 degrees with the Meridians. In this case, the component of the earth's velocity field of the HQS adds $6.716 \mathrm{~km} / \mathrm{sec}$ to the presumed light velocity in vacuum. This is almost exactly the excess velocity found by the neutrinos team from CERN and gives an effective velocity of $c^{\prime}=299799174 \mathrm{~m} / \mathrm{sec}$. Therefore, the neutrinos are predicted to reach the OPERA Lab $\sim 55 \mathrm{~ns}$ earlier than presumed for light by the current theories. This however, does not break the light postulate. Light, along the same path (in vacuum) too would reach $\sim 55$ ns too early to the OPERA Lab. 
In 2011 neutrinos from CERN were announced to reach too early to the OPERA Lab by very nearly $60 \mathrm{~ns}$. This however was interpreted as a faster than light velocity, without any reasonable justification [23]. Later the data were withdrawn because of conflict with the light postulate [24]. From the view of the present work, this too early arrival must be correct and does not break the light postulate.

Actually this affair is in standby, waiting for a final verdict. New experiments are in course, also in the USA, in which neutrinos will be shut from the Fermilab (Chicago) to Stanford (South Dakota), distant $1300 \mathrm{~km}$ toward the West. These neutrinos thus will speed in the opposite sense of the earth's Keplerian velocity field of the HQS that creates the earth's gravitational field. In this case the neutrinos are predicted to reach Stanford more than 115 ns too late. However, light along the same path (in vacuum) too is predicted to reach too late by the same 115 ns.

If the predicted one-way anisotropy of the neutrinos is confirmed, this experiment, besides the many others, described above in the previous Sections, will turn the HQS dynamics an incontestable reality and constitute it into a top scientific achievement, rivaling in relevance with the discovery of the heliocentric system.

\subsubsection{The Astronomical Motions Closely Track the Motion of the HQS throughout the Universe}

The planetary orbits lie all closely within the equatorial plane of the solar Keplerian velocity field of the HQS and the orbit of the solar system lies closely within the equatorial plane of the galactic velocity field of the HQS. This orbital configuration minimizes the velocity of the astronomical bodies with respect to the local HQS. Wavelength stretching during the concomitant expansion of the HQS and of the universe reduces the kinetic energy of particles and the energy of radiation with respect to the local HQS, which is well demonstrated by the cosmic microwave background radiation. Moreover, the averaging down of random velocity, during gravitational agglomeration of matter into large matter bodies too reduces the velocity, momentum and kinetic energy of the particles with respect to the local HQS, converting it in heat. Visibly not only the planets of the solar system, not only the solar system in the Milky-Way galaxy, however astronomical bodies in general throughout the universe are very closely stationary with respect to the local HQS in the respective gravitational fields. This predicts the universality of the laws of physics.

\subsubsection{Gravitational Waves}

The occurrence of gravitational waves was predicted by Einstein's GR. HQS dynamics predicts that binary systems naturally emit gravitational waves. However, the amplitude and frequency of usual binary stars are very low. Binary merges of neutron stars and black-holes however generate amplitudes and frequencies that are observable. In the black-holes the velocity of the HQS in the velocity fields $(G M / r)^{1 / 2}$, creating the respective gravitational fields, achieves the velocity of 
light. In 2016, one century after Einstein's prediction, the occurrence of gravitational waves, generated by the merge of a black-hole binary, finally confirmed Einstein's prediction [25].

\section{Final Comments and Conclusions.}

The HQS is a macroscopic quantum state of bosons, ruled by the principles of quantum physics. It plays a fundamental role in the microscopic quantum world giving mass and ruling the motion of the elementary particles. It plays a fundamental role in the macroscopic world of gravity too, where a Keplerian velocity field of the HQS Equation (5) implements the outside-inside spherical centrifuge mechanism of gravity that creates the centrifugal effects pulling matter toward the gravitational centers. This ingenious centrifuge mechanism governs the gravitational dynamics on earth, in the solar system and throughout the universe. It too predicts the non-Keplerian galactic gravitational dynamics, without the need of dark matter [26]. The HQS dynamics opens the way to the unification of all the force fields. In the scenario of the HQS dynamics, the astronomical bodies, throughout the universe, are all very closely stationary with the local HQS in the velocity fields creating the respective gravitational fields, which predicts the universality of the laws of physics. The macro physics of the HQS too governs the global dynamics, the accelerated expansion of the universe [12]. Without the presence of the HQS, the matter universe simply would not exist. The HQS literally creates and governs our universe.

\section{Conflicts of Interest}

The author declares no conflicts of interest regarding the publication of this paper.

\section{References}

[1] Laue, M.V. (1955) Annalen der Physik, 38.

[2] Lorentz, H.A., Einstein, A., et al. (1923) The Principle of Relativity. Dover Publications, New York.

[3] Ashby, N. (1996) Mercury, 23-27.

[4] Bailey, H., Borer, K., Combley, F., Drumm, H. and Krienen, F. (1977) Nature, 268, 301-305. https://doi.org/10.1038/268301a0

[5] Anderson, P.W. (1963) Physical Review Journals Archive, 130, 439. https://doi.org/10.1103/PhysRev.130.439

[6] Meissner, W. and Ochsenfeld, R. (1933) Naturwissenschaften, 21, 787-788. https://doi.org/10.1007/BF01504252

[7] Ginzburg, V.L. and Landau, L.D. (1950) Journal of Experimental and Theoretical Physics (JETP), 20, 1064.

[8] Higgs, P.W. (1964) Physical Review Letters, 13, 508. https://doi.org/10.1103/PhysRevLett.13.508

[9] Englert, F. and Brout, R. (1964) Physical Review Letters, 13, 321. https://doi.org/10.1103/PhysRevLett.13.321

[10] Carrol, S.M. (2000) arXiv:astro-ph/0004075v 
[11] Sola, J. (2013) arXiv:1306.1527v3 [gr-qc]

[12] Schaf, J. (2017) Int. J. of Adv. Res. in Phys. Sc., 4, 1.

[13] Hatch, R.R. (2004) GPS Solutions, 8, 67-73. https://doi.org/10.1007/s10291-004-0092-8

[14] Hatch, R.R. (2004) Foundations of Physics, 34, 1725-1739. https://doi.org/10.1007/s10701-004-1313-2

[15] Hatch, R.R. (2007) Physics Essays, 20, 83-100. https://doi.org/10.4006/1.3073811

[16] Schaf, J. (2018) Journal of Modern Physics, 9, 1111-1143. https://doi.org/10.4236/jmp.2018.95068

[17] Schaf, J. (2018) Journal of Modern Physics, 9, 395-418. https://doi.org/10.4236/jmp.2018.93028

[18] Schaf, J. (2018) Journal of Modern Physics, 9, 2125-2134. https://doi.org/10.4236/jmp.2018.912133

[19] Miller, D.C. (1933) Review of Modern Physics, 5, 203. https://doi.org/10.1103/RevModPhys.5.203

[20] Shapiro, I.I., et al. (1971) Physical Review Letters, 26, 1132. https://doi.org/10.1103/PhysRevLett.26.1132

[21] Pound, R.V. and Snider, J.L. (1965) Physical Review B, 140, B788. https://doi.org/10.1103/PhysRev.140.B788

[22] Brault, J.W. (1963) Bulletin of the American Physical Society, 8, 28.

[23] Adam, T., et al. (2011) arXiv:1109.4897v2 [hep-ex].

[24] Adam, T., et al. (2012) arXiv:1109.4897v4 [hep-ex].

[25] Abbott, B.P., et al. (2016) Physical Review Letters, 116, Article ID: 061102. https://doi.org/10.1103/PhysRevLett.116.061102

[26] Schaf, J. (2018) Journal of Modern Physics, 9, 1883-1905. https://doi.org/10.4236/jmp.2018.910119 